\title{
Fully Anharmonic Resonance Raman Spectrum of Diatomic Molecules Through Numerical Solution of the Vibrational Schrodinger Equation
}

\author{
Gustavo J. Costa, Rogério Custodio, Pedro A. M. Vazquez \& Luciano N. Vidal
}

\section{Introduction}

The molecular contribution to the intensity of the Raman scattering is given by the general polarizability $\alpha_{\rho \sigma}{ }^{\text {fi }}$. In the far-from-resonance (FFR) regime, the energy of the excitation radiation $\left(\hbar \omega_{0}\right)$ is far from that of any electronic transition and $\alpha_{\rho \sigma}{ }^{\text {fi }}$ receives balanced contributions of many excited electronic states, expressed by the well known Placzek's electronic polarizability, whose geometrical derivatives are related to the intensities of FFR Raman. ${ }^{1}$ On the other hand, the Resonance Raman (RR) scattering occurs when $\hbar \omega_{0}$ is close from the energy of an electronic transition. In RR the intensity of the scattered radiation is orders of magnitude stronger than that of FFR regime and $\alpha_{\rho \sigma}{ }^{\text {fi }}$ is dominated by one (or few) excited electronic state. ${ }^{1}$ Therefore, this form of Raman provides structural and dynamical information of both ground and excited (resonance) electronic states. Within the Born-Oppenheimer (BO) and Frank-Codon (FC) approximations, the corresponding expression for $\alpha_{\rho \sigma}{ }^{\text {fi }}$ of $\mathrm{RR}$ is given by:

$$
\alpha_{\rho \sigma}^{f i}=\frac{1}{\hbar}\left\langle g\left|\mu_{\rho}\right| e\right\rangle\left\langle e\left|\mu_{\sigma}\right| g\right\rangle \sum_{n} \frac{\left\langle v_{f} \mid n\right\rangle\left\langle n \mid v_{i}\right\rangle}{\omega_{e g}+\omega_{n i}-\omega_{0}+i \Gamma_{e}}
$$

This expression involves, among other quantities, FC integrals of the initial $\left(\mathrm{v}_{\mathrm{i}}\right)$ and final $\left(\mathrm{v}_{\mathrm{f}}\right)$ vibrational states in the ground electronic state $g$ with vibrational states (n) of the resonance electronic state e. In order to simplify the calculation of FC integrals, the Potential Energy Surface (PES) of $g$ and e states are considered harmonic and those integrals are evaluated analytically from recurrence formulas. ${ }^{2}$ To the best of our knowledge, there is only one article reporting the calculation of FC integrals for anharmonic wave-functions in RR. ${ }^{3}$ On that pure theoretical work, the RR cross section of diatomic systems is obtained from analytic integration of unidimentional eigenfunctions of the Morse potential. The FC factors were computed for several values of the vibrational quantum numbers $\mathrm{v}$ and $\mathrm{n}$ of hypothetical molecules, and were compared with those from analytic solution of the harmonic potential. It was shown that FC factors differ only few percent between Morse and harmonic models for $\mathrm{v}, \mathrm{n}=0,1$ or 2 , however, for higher values the anharmonic FC factors can be many time greater or lower than the corresponding harmonic data. In the case of RR cross sections, the anharmonic effects can be strong even for fundamental transitions.

In the present study, the FC integrals are numerically evaluated for vibrational states of the true PES obtained from correlated ab initio methods. The vibrational wave-functions are calculated using a version of the Variational Monte Carlo (VMC) 
method. Our method is applied to calculate the FC RR spectrum of diatomic molecules. In addition, the Herzberg-Teller (HT) intensity borrowing mechanism is also taken into account in the current model, thus $\alpha_{\rho \sigma}{ }_{\rho i}$ can be corrected for the effect of coupling the electronic transition moments with the nuclear motion.

\section{Methods}

The electronic structure calculations were performed with Dalton quantum chemistry code4 for a single reference (e.g. CCSD) and multi-reference (e.g. NEVPT2) electron correlation $a b$ initio methods using correlation consistent basis sets augmented with diffuse functions (aug-cc-pVXZ, X=D,T,Q). The electric dipole transition moment $\left\langle g\left|\mu \_\rho\right| e\right\rangle$ and vertical excitation energy $\hbar \omega \_$eq were obtained from the Linear Response of the CCSD wave-function at the ground state equilibrium geometry. HT effects can also be taken into account in our model if the electronic transition moment is expanded in Taylor series of normal coordinate Q:

$$
\left\langle g\left|\mu_{\rho}\right| e\right\rangle=\left\langle g\left|\mu_{\rho}\right| e\right\rangle_{0}+\left(\frac{d\left\langle g\left|\mu_{\rho}\right| e\right\rangle}{d Q}\right)_{0} Q+\cdots
$$

When the above expansion is used for $\langle\mathrm{g}|\mu \rho \rho| \mathrm{e}\rangle$ , the resulting $\alpha_{\rho \sigma}{ }^{\mathrm{fi}}$ demands the computation of FC and HT integrals of the general form: $\left\langle v_{f}\left|Q^{m}\right| n\right\rangle$ and $\left\langle n\left|Q_{m}\right| v_{i}\right\rangle$, with $\mathrm{m}=0,1,2 \ldots$. All FC and HT integrals were evaluated by numerical methods. The required geometric derivatives of the electronic transition moments were computed at the ground state equilibrium geometry using central difference formulas. These derivatives, the FC and HT integrals and the RR spectrum were obtained by our code PLACZEK, developed for the calculation of infrared and Raman spectra.5 Temperatures effects may be important for those systems with low energy vibrations. In such cases, the RR cross sections of several initial v_i states are calculated and average by their thermal population. From the current approach, any RR fundamental or overtone transition is available. They have quite similar computational costs and depend on the speed of convergence of the cross section with respect to the number of intermediate vibrational states $\mathrm{n}$.

The vibrational analysis was carried out considering an alternative Quantum Monte Carlo. In this formalism the Hamiltonian is represented as the combination of the well-know kinetic energy operator and the potential energy is selected according to the requirements of system. In this work, the potential energy was taken from ab initio calculations described above. The second derivative of the Laplacian is describe by a central difference expression:

$$
\left(\frac{\partial \psi}{\partial x}\right)=\left(\frac{2}{h_{i}+h_{i-1}}\right)\left(\frac{\psi_{i+1}-\psi_{i}}{h_{i}}-\frac{\psi_{i}-\psi_{i-1}}{h_{i-1}}\right)
$$

where, $h_{i}=\chi_{i+1}-\chi_{i}$. The initial wave function is described as a set of arbitrary values defined in each particular discretized coordinate. From the discretized mean value theorem, the Hamiltonian and the discretized wave function it is possible to estimate the total energy of the system.6 One of the discrete values of the wave function is selected at random and modified by, for example: $\psi_{\text {new }}=\psi_{\text {old }}+(0.5$-rand $) \delta$, where rand is a uniform distributed random number and is a parameter defining the degree of change in the wave function. From the modified wave function, the total energy is calculated and compared with the precious energy. If the new energy is accepted, the modification in the wave function is preserved. If the new energy increases, the change in the wave function is rejected. The procedure follows the variational principle and the final energy is achieved after a selected degree of convergence of the system is defined.

\section{Results and Discussion}

Preliminary results are presented in Figure 1, showing some vibrational wave-functions and their energies for $\mathrm{N} 2$ evaluated at the ground electronic state. The PES was calculated at the NEVPT2 $(6,6)$ /aug-cc-pVTZ level. The spectroscopic constants obtained for different sets of vibrational states are shown in Table 1 together with experimental data. They are useful to access the quality of the vibrational wave-functions. 

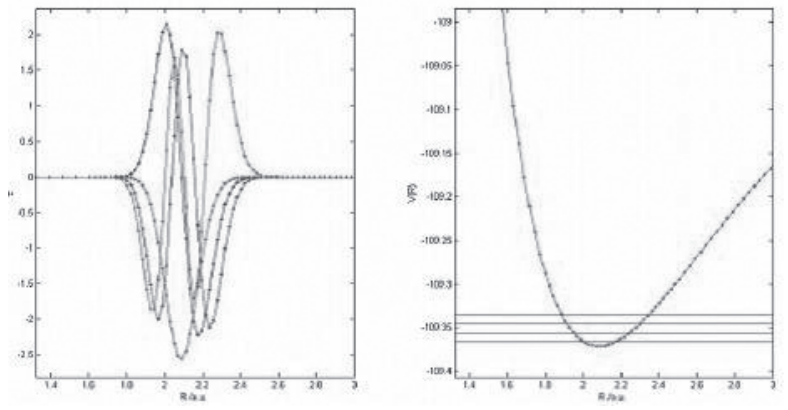

Figure 1. Anharmonic vibrational wave-functions (left) and their energy (right) for the first 4 states of N2.

Table 1. Spectroscopic constants calculated for the ground electronic state of N2 at the NEVPT2(6,6)/aug-cc-pVTZ level for several vibrational states $(\mathrm{v})$.

\begin{tabular}{|c|c|c|c|}
\hline $\mathbf{v}$ & 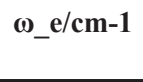 & $\underset{\text { cm-1 }}{\omega_{\text {end }} \text { e } X_{-} \text {e/ }}$ & $\underset{\text { cm-1 }}{\omega_{\text {ende }} \text { e } /}$ \\
\hline 5 & 2344.903 & 23.335 & 0.157 \\
\hline 8 & 2346.754 & 24.503 & 0.347 \\
\hline 10 & 2323.642 & 16.111 & -0.409 \\
\hline 12 & 2349.555 & 23.922 & 0.155 \\
\hline 15 & 2344.457 & 22.196 & 0.020 \\
\hline Exp.7 & 2358.57 & 14.324 & -0.00226 \\
\hline
\end{tabular}

\section{Conclusions}

Our method is under final development with the VMC code ready and RR results now being compared with analytical data from the harmonic model to check their accuracy.

\section{Acknowledgments}

The authors are grateful for the support given from the CNPQ, FAPESP e FAEPEX-UNICAMP.

\section{References}

1. D. A. Long, The Raman Effect; John Wiley \& Sons, LTD:

Chichester, (2002).

2. F. Santoro, C. Cappelli and V. Barone, J. Chem. Theory Comput., 7, 1824, (2011). F. Egidi, J. Bloino, C. Cappelli and V. Barone, J. Chem. Theory Comput., 10, 346, (2014). F. Santoro, R. Improta, A. Lami, J. Bloino, and V. Barone, J. Chem Phys., 126, 084509, (2007).
3. H. Kono, Z. Z. Ho and S. H. Lin, J. Chem. Phys. 80, 1760, (1984).

4. Dalton, a molecular electronic structure program, Release Dalton2015. (2015), see http://daltonprogram.org.

5. O. Beruski and L. N. Vidal, J. Comput. Chem., 35, 290, (2014). L. N. Vidal and P.A. M. Vazquez, Quím. Nova, 35, 2076 (2003).

6. R. Custodio, M. R. Custodio, E. J. Creatto, Quim. Nova, 35, 2076 (2014).

7. K. Huber and G. Herzberg, Molecular Spectra and Molecular Structure. IV. Constants of Diatomic Molecules, 2nd ed., Van Nostrand Reinhold: New York, (1979).

\section{Gustavo Juliani Costa ${ }^{a}$, Rogério Custodio ${ }^{b}$, Pedro A. M. Vazquez ${ }^{\text {b }}$ \& Luciano Nassif Vidal ${ }^{a}$}

${ }^{a}$ Universidade Tecnológica Federal do Paraná, DAQBI, Curitiba/PR, Brazil.

${ }^{\mathrm{b}}$ Universidade Estadual de Campinas, IQ-DFQ, Campinas/SP, Brasil. 